\title{
NOTES ON THE SPECTRUM OF $\gamma$ ARGUS
}

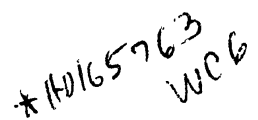

On examining three three-prism plates of $\gamma$ Argus taken by the D. O. Mills Expedition at Santiago, Chile, with reference to the character and place of the WolfRayet band at $4652 \mathrm{~A}$, which is represented by or at least included in the broad bright band extending from about $4640 \mathrm{~A}$ to $4670 \mathrm{~A}$, I noticed a faint indication of a break or division in the band at about 4655A. This appearance, however, could as easily be interpreted as a faint, narrow absorption line, particularly in view of the existence of a line at $4654.736 \mathrm{~A}$ in the iron spectrum. But narrow iron lines have not thus far been observed in Class $O$ spectra. It occurred to me that this broad band might be composed of two overlapping bands corresponding to the effective wave-lengths $4652 \mathrm{~A}$ and $4658 \mathrm{~A}$, which are now known to be represented in Wolf-Rayet spectra. One of the three plates was therefore measured and the following results obtained for the broad band and the neighboring band at $4688 \mathrm{~A}$.

\begin{tabular}{|c|c|c|c|}
\hline $\begin{array}{l}\text { Bright } \\
\text { bands }\end{array}$ & $\begin{array}{c}\text { Measured } \\
\text { wave-lengths }\end{array}$ & $\begin{array}{l}\text { Middle } \\
\text { of band }\end{array}$ & Width \\
\hline Violet side & 4641A) & $4648 \mathrm{~A}$ & $14 \mathrm{~A}+$ \\
\hline Division & 4655 & & $30 \mathrm{~A} \pm$ \\
\hline Red side & 4671. & 4663 & $16 \mathrm{~A}+$ \\
\hline Violet side & 4679 & & \\
\hline Red side & 4698 & 4688 & $19 \mathrm{~A}+$ \\
\hline
\end{tabular}

The widths given in the above table must not be taken as definite or exact. If the 30 -angstrom band is composed of two overlapping bands, the widths of the latter must be greater than the widths of the divisions as noted in the table.

These bands have no defined edges, but shade off over intervals of several angstroms, so that it is difficult to designate beginning, ending, or mid-point. The values in the third column are the means of those in the second. The mean values $4648 \mathrm{~A}$ and $4663 \mathrm{~A}$ do not correspond to any Wolf-Rayet bands thus far observed. But if this broad band of $\gamma$ Argus is composed of two overlapping bands, then their mid-points would be closer together than indicated by the values 4648 and 4663 , and the effective wave-length of the violet component would be greater than $4648 \mathrm{~A}$ and that of the red component less than 4663A. Thus it would not be impossible for this broad band to consist of the two Walf-Rayet bands known as 4652A and 4658A.

An extension of the idea of complex or merging bands would be that this is the nature or explanation of others in other Wolf-Rayet stars, especially those in this same region, some of which are one hundred or more angstroms wide, extending from about $4600 \mathrm{~A}$ to $4700 \mathrm{~A}$; for example, that in the star SDM $-21^{\circ} \cdot \mathrm{k}$ $4864\left(18^{\mathrm{h}} 2^{\mathrm{m}} 5\right)$. In this region of spectrum are known at least six Wolf-Rayet bands, namely, 4615, 4626, $4633,4652,4658,4688$. The investigation of these extended bands with various dispersions and varied exposures and otherwise would probably lead to interesting results.

Upon referring to Dr. Campbell's observations of $\gamma$ Argus, made with single-prism dispersion in 1893, it is found that he distinguished two maxima in the broad band extending from $4640 \mathrm{~A}$ to $4670 \mathrm{~A}$, which caused him to say in his published description of the spectrum of $\gamma$ Argus:" "The broad band 4651 is strongly suspected to be double, with components near 4643 and 4659.'" He has not noted the double appearance of this band in the spectrum of any other Wolf-Rayet star. ${ }^{2}$ Dr. Campbell's values 4643, 4651, 4659 , for the mid-points of the whole band and of its divisions, are thus systematically four angstroms less than those in the table above. The detection of the apparent division in this band on both one- and threeprism plates gives it a certain amount of probabilty of existence, whatever its cause or explanation may be.

The bright band at $4688 \mathrm{~A}$ is described in the Harvard Annals" as follows: "A narrow dark line is superposed on this bright band" ; "the bright bands 4682 to 4700 , . . are reversed." Neither of these effects is discernible on our three-prism plates. In fact, these plates show no features except the two bright bands noted in the table above. The continuous spectrum is almost uniform except for an extremely slight and extended absorption in the regions of $\mathrm{H}_{\gamma}$ and $\mathrm{H} \delta$. Campbell's observations ${ }^{4}$ and those at Harvard Observatory ${ }^{3}$ indicate narrow dark lines across the bright $\mathrm{H} \beta$ band and others.

There has recently appeared in Monthly Notices ${ }^{5}$ an article by $\mathrm{Mr}$. W. M. Worssell of the Union Observatory, Johannesburg, South Africa, upon the spectrum of $\gamma$ Argus, containing a partial description of his work with the Franklin-Adams prismatic camera, and the results of a study by Professor Fowler. of South Kensington, England, of one of Mr. Worssell's plates of $\gamma$ Argus. The plates were exposed sufficiently to obtain the less refrangible part of the spectrum, thus overexposing the blue region. A table

\footnotetext{
1 Astr. and Astroph., 12, 555, 1893.

2 Astr. and Astroph., 13, 455 to 467, 1894.

3 Annals H. C. O., 28, 246, 247, 1901.

4 Astr. and Astroph., 13, 457, 1894.

5 Mon. Not. R. A. S., 76, 418, 1916.
} 
Is given of twenty-one lines or bands found in the :region $6700 \mathrm{~A}$ to $4862 \mathrm{~A}$. A few identifications are ionade, namely, for hydrogen, helium and possibly int earbon; but the particular point of interest there is the finding of ten or eleven instances of reriviversal, four strong or definite and six or seven faint or suspected. The reversals of helium $\mathrm{D}_{3}$ and hydrogen $\mathrm{H} \beta$ are the most conspicuous. No mention is made of reversal being seen in the two bright bands at $4686 \mathrm{~A}$ and $4652 \mathrm{~A}$ which are conspicuous in the overexposed region of the spectrum. However, in view of the evidence of reversal found by Worssell and Fowler, it is very possible that the division seen on our Southern Mills plates in the band $4640 \mathrm{~A}$ to $4670 \mathrm{~A}$ may be a reversal of the band $4652 \mathrm{~A}$ if this be the effective wave-length of the whole band.

Recent one-prism plates of $\gamma$ Argus taken at Santiago, Chile, by Wilson and Scott give indication that the absorption lines at $\mathrm{H} \delta, \mathrm{H}_{\gamma}$ and $\mathrm{He} 4471$ are bordered by slight brightening of the continuous spectrum. This accords with the description given by Harvard Observatory, ${ }^{6}$ and may place these lines in the class of reversed lines or bands. Mr. Worssell, however, did not detect any brightening on his plates, saying that the hydrogen lines to violet of $\mathrm{H} \beta$ appear to be wholly dark. There is also indication that the reversal increases in strength toward the violet, which property would associate the spectrum of $\gamma$ Argus with the spectra of the bright-line stars of Class B. ${ }^{7}$ On the Santiago one-prism plates bright helium 4922A and $4713 \mathrm{~A}$ are not perceptible; also on the bright helium 5015A the reversal, "feeble" according to Mr. Worssell, is not discernible. The bright band 4442A is strong on these plates. Hydrogen and helium absorption bands are conspicuous in the violet, namely, at $3875 \mathrm{~A}$ (unknown), 3888A ( $\mathrm{He}), 3889 \mathrm{~A}(\mathrm{H} \zeta)$, $3970 \mathrm{~A}(\mathrm{H} \epsilon)$, and $4026 \mathrm{~A}(\mathrm{He})$. In fact, the whole stretch of spectrum presented on these plates shows a wavy intensity.

Further observation and study are quite likely to bring out critical data or facts concerning this peculiar spectrum.

G. F. PADDOCK.
June, 1916.

Issued October 21, 1916.

B Annals H. C. O., 28, 247, 1901.

? Campbell, Ap. Jour., 2, 177, 1895; Merrill, Lick Obs. Bull., 7, 175, 1913. 